\title{
ON FRACTIONAL INTEGRALS OF PURE IMAGINARY ORDER IN $L_{p}$
}

G. K. KALISCH ${ }^{1}$

The purpose of this paper is to extend some results of Kober [4] from $L_{2}$ to $L_{p}, 1<p<\infty$. Sahnovič [7], using a similar method, obtained a result similar to Kober's (in $L_{2}$ ). His purpose was to show the similarity of the operators $M$ and $M+i V$ in $L_{2}(0,1)$ where $(M f)(x)=x f(x)$ and $(V f)(x)=\int_{0}^{x} f(y) d y$; he found that the operator $P$ implementing this similarity, that is, a bounded operator $P$ with bounded inverse $P^{-1}$ such that $P^{-1} M P=M+i V$, is precisely $P=J^{i}$ where $J^{5}$ is the operator of fractional integration

$$
\left(J^{\zeta} f\right)(s)=(1 / \Gamma(\zeta)) \int_{0}^{s}(s-t)^{\zeta-1} f(t) d t
$$

where $\zeta=\xi+i \eta$ is a complex variable with real $\xi$ and $\eta$ and $f$ and $J 5 f$ (if it exists) are functions defined on the interval $[0,1]$. Our motivation is similar to Sahnovič's; we are publishing elsewhere [3] the application of our present results to the type of problem considered by him except that we omit the restriction $p=2$. We are using without further reference results and notations from the exposition by Hille and Phillips [2] of Kober's results such as the boundedness and the strongly continuous holomorphic semigroup character of $J^{5}$ for $\xi>0$.

For this semigroup we prove the conclusions of Theorems 17.9.1 and 17.9.2 in Hille and Phillips.

TheOREM G. The holomorphic semigroup $\left\{J^{5}\right\}$ with $\xi>0$ defined in $L_{p}=L_{p}(0,1)$ with $1<p<\infty$ admits a bounded boundary group $\left\{J^{i \eta}\right\}$ in the sense that the $L_{p}$-limit

$$
\lim _{\xi \rightarrow 0+} J^{\xi+i \eta} f=J^{i \eta} f
$$

exists for all $f \in L_{p}$. The set $\left\{J^{i \beta}\right\}$ for all real $\beta$ is a strongly continuous group of bounded operators, $J^{i \beta}$ commutes with $J^{5}$ for all $\xi>0$ and all real $\beta$ and $\eta$ and

$$
J^{\xi+i \eta}=J^{\xi} J^{i \eta} .
$$

Received by the editors December 3, 1965.

1 This research was supported in part by a grant from the National Science Foundation, hereby gratefully acknowledged. 
If $A$ is the infinitesimal generator of $\left\{J^{\xi}\right\}$ then iA is the infinitesimal generator of $\left\{J^{i \beta}\right\}$.

The proof will be accomplished if the hypothesis of theorems 17.9.1 and 17.9.2 in Hille and Phillips is verified. This is done in the following theorem.

Theorem B. Let $\xi \in(0,1]$ and $\eta \in[-1,1]$. Then there exists a finite positive constant $M$ independent of $\xi$ and $\eta$ but dependent on $p \in(1, \infty)$ such that the $L_{p}$-norm of $J^{5}$ satisfies

$$
\|J \xi\|_{p}<M \text {. }
$$

The proof is similar to the proofs by Kober and Sahnovič; the missing ingredient is Mihlin's extension to Fourier integrals in $L_{p}$ [6] of Marcinkiewicz's result on multipliers of Fourier series in $L_{p}$ [5]; see also Zygmund [8].

Write

$$
\begin{aligned}
g(s) & =\left(J^{\zeta} f\right)(s)=(d / d s)\left(J^{\zeta+1} f\right)(s) \\
& =\frac{1}{\Gamma(\zeta+1)} \frac{d}{d s}\left(\int_{0}^{s}(s-t)^{\zeta} f(t) d t\right)
\end{aligned}
$$

for $f \in L_{p}(0,1)$ and $\xi>0$. We shall use the following substitutions: $s=e^{\sigma}, t=e^{\tau}, \phi(\tau)=e^{(\tau / p)} f\left(e^{\tau}\right), \gamma(\sigma)=e^{(\sigma / p)} g\left(e^{\sigma}\right)$. If $f$ and $g$ are in $L_{p}(0,1)$, then $\phi$ and $\gamma$ are in $L_{p}(-\infty, 0)$ and conversely, and we have $\|f\|_{p}$ $=\|\phi\|_{p}$ and $\|g\|_{p}=\|\gamma\|_{p}$. We then obtain

$$
\gamma(\sigma)=\frac{e^{-a \sigma}}{\Gamma(\zeta+1)} \frac{d}{d \sigma}\left(\int_{-\infty}^{\sigma}\left(e^{\sigma}-e^{\tau}\right)^{\zeta} \phi(\tau) e^{a \tau} d \tau\right)
$$

where $(1 / p)+a=1$. Write

$$
\phi(\tau)=\left(\frac{1}{(2 \pi)^{1 / 2}}\right) \int_{-\infty}^{\infty} e^{-i \tau \beta} \Phi(\beta) d \beta .
$$

We now confine ourselves to a set of functions $f$ such that (2) is valid with corresponding $\Phi$ in $L_{1}(-\infty, \infty)$; this set is dense in $L_{p}(0,1)$. After substituting (2) into (1) and interchanging the order of integration, we can evaluate the inner integral and obtain

$$
\begin{aligned}
\gamma(\sigma) & =e^{-a \sigma} \frac{d}{d \sigma}\left[\left(\frac{1}{(2 \pi)^{1 / 2}}\right) \int_{-\infty}^{\infty} e^{\sigma(\zeta+a-i \beta)} \frac{\Gamma(a-i \beta)}{\Gamma(\zeta+1+a-i \beta)} \Phi(\beta) d \beta\right] \\
& =\left(\frac{e^{2 \sigma \zeta}}{(2 \pi)^{1 / 2}}\right) \int_{-\infty}^{\infty} e^{-i \sigma \beta} \frac{\Gamma(a-i \beta)}{\Gamma(\zeta+a-i \beta)} \Phi(\beta) d \beta .
\end{aligned}
$$


We show below that $\Psi(\beta)=(\Gamma(a-i \beta) / \Gamma(\zeta+a-i \beta))$ is bounded in absolute value uniformly with respect to $\zeta$ in the range considered in the theorem; this shows that our hypothesis on $\Phi$ allows us to differentiate under the integral sign. We now restrict $f$ again so that $\phi \in L_{1}(-\infty, 0)$; this still leaves us with a set of $f^{\prime}$ 's dense in $L_{p}(0,1)$. This last restriction allows us to write

$$
\Phi(\beta)=\frac{1}{(2 \pi)^{1 / 2}} \int_{-\infty}^{\infty} e^{i \alpha \beta} \phi(\alpha) d \alpha
$$

so that

$$
\gamma(\sigma)=e^{\sigma \zeta} \int_{-\infty}^{\infty} e^{-i \beta \sigma} \Psi(\beta) \int_{-\infty}^{\infty} e^{i \alpha \beta} \phi(\alpha) d \alpha d \beta
$$

If now there exists a constant $M$ independent of $\beta$ and $\zeta$ (in our range) and $\phi$ such that

$$
|\Psi(\beta)|<M, \quad\left|\beta \Psi^{\prime}(\beta)\right|<M
$$

for all real $\beta$ then we can apply Mihlin's theorem and conclude that $\|\gamma\|_{p}<M\|\phi\|_{p}$ or $\|g\|_{p}=\|J f f\|_{p}<M\|f\|_{p}$ which is the desired conclusion for a set of $f$ 's dense in $L_{p}(0,1)$ and hence for all $f$ in $L_{p}(0,1)$.

The two inequalities (3) are simple consequences of Stirling's formula for $\Gamma(z)$ and $\log \Gamma(z)$ as found for example in Franklin [1] since it is clearly sufficient to check them for large $|\beta|$.

Thus for the first inequality (3), we have the asymptotic relation for large $|\beta|$

$$
\begin{aligned}
|\Psi(\beta)| \sim & \exp (\xi)|\beta|^{-\xi} \exp [\eta \arg (\zeta+a-i \beta)] \\
& \cdot \exp (-\beta[\arg (a-i \beta)-\arg (\zeta+a-i \beta)]) .
\end{aligned}
$$

The angles arg . . . are comprised between $-\pi / 2$ and $\pi / 2$. On checking the right side of the above asymptotic relation, we see that it is bounded by $\exp (2+\pi / 2)$ and that its limit as $|\beta| \rightarrow \infty$ is zero.

For the second inequality (3), we have

$$
\beta \Psi^{\prime}(\beta)=\Psi(\beta) \cdot \beta[((d / d z) \log \Gamma)(a-i \beta)-((d / d z) \log \Gamma)(\zeta+a-i \beta)] .
$$

We know that the first factor is bounded in absolute value. The boundedness of the second factor follows from the formula [1]

$\log \Gamma(z)=-z+\left(z-\frac{1}{2}\right) \log z+\log (2 \pi)^{1 / 2}+\left(\frac{1}{12 z}\right)-\int_{0}^{\infty} \frac{P_{2}(x)}{(z+x)^{2}} d x$ 


\section{REFERENCES}

1. Philip Franklin, $A$ treatise on advanced calculus, Wiley, New York, 1940.

2. Einar Hille and Ralph S. Phillips, Functional analysis and semigroups, Amer. Math. Soc. Colloq. Publ., Vol. 31, Amer. Math. Soc., Providence, R. I., 1957.

3. G. K. Kalisch, Similarity invariants of a certain class of operators, (to appear).

4. H. Kober, On a theorem of Schur and on fractional integrals of purely imaginary order, Trans. Amer. Math. Soc. 50 (1941), 160-174.

5. J. Macinkiewicz, Sur les multiplicateurs des séries de Fourier, Studia Math. 8 (1939), 78-91.

6. S. G. Mihlin, Integraly Fur'e i kratnye singuljarnye integraly, Vestnik Leningrad. Univ. Ser. Mat. Meh. Astronom. 1957, no. 7, 143-155.

7. L. A. Sahnovix, Privedenie odnogo nesamosoprjažennogo operatora s nepreryvnym spektrom k diagonal'nomu vidu, Uspehi Mat. Nauk 13 (1958), no. 4 (42), 193-196.

8. Antoni Zygmund, Trigonometric series, Cambridge Univ. Press, New York, 1959.

UNIVERSITY OF MINNESOTA AND

UNIVERSITY OF CALIFORNIA, IRvine 